УДК 536.468

\title{
ОБОСНОВАНИЕ РЕСУРСОЭФФЕКТИВНОСТИ ТЕХНОЛОГИЙ СЖИГАНИЯ ВОДОУГОЛЬНЫХ ТОПЛИВ С ДОБАВКАМИ БИОМАССЫ
}

\author{
Малышев Дмитрий Юрьевич1, \\ dmitry.mlv@gmail.com

\section{Сыродой Семен Владимирович1,} \\ ssyrodoy@yandex.ru \\ ${ }^{1}$ Национальный исследовательский Томский политехнический университет,
Россия, 634050, г. Томск, пр. Ленина, 30.
}

\begin{abstract}
Одной из наиболее актуальных проблем современности является экологическая безопасность. Загрязнение атмосфреры Земли в последние десятилетия обусловлено в значительной степени работой тепловых электрических станций, сжигающих уголь, на территориях наиболее развитых государств (США, Китай, Индия и др.). Использование нового класса топлива - био-водоугольного - может позволить существенно снизить выбросы антропогенных газов, образующихся при сжигании уелей в топках паровых и водогрейных котлов, в атмосфреру Земли, расширить сырьевую базу современных тепловых электрических станций и повысить в целом ресурсоэффрективность угольной теплоэнергетики.

Цель: исследование влияния концентрации древесной биомассы в жидких композиционных топливах на интегральные характеристики процесса зажигания капель био-водоугольных топлив в условиях высокотемпературного нагрева.

Объект: водоугольная суспензия на основе угля марки $T$, воды и еловой хвои. Экспериментальные исследования проведены при следующих массовых концентрациях уәля и биомассы: 50/0 \%, 45/5%, 40/10 \%, 35/15 \%, соответственно (50 \% - вода).

Mетод: экспериментальное определение с использованием высокоскоростной видеокамеры Photron FASTCAM CA4 временных характеристик процессов зажигания капель био-водоугольных суспензий в условиях, соответствующих по интенсивности нагрева камерам сгорания паровых и водогрейных котлов; регистрация температуры среды с использованием хромельалюмелевых термопар.

Результаты. Установлено влияние массовой концентрации лесного горючего материала на времена задержки зажигания (tign) водоугольных топлив. Показано, что при содержании в топливной композиции 15 \% биомассы времена задержки зажигания уменьшаются более чем в три раза по сравнению с водоугольным топливом без добавления биомассы при относительно низких температурах топочной среды. Результаты выполненных экспериментальных исследований также являются базой для развития моделей горения жидких композиционных топлив.
\end{abstract}

\section{Ключевые слова:}

Био-водоугольное топливо, биомасса, лесной горючий материал, зажигание, время задержки зажигания, эксперимент, энергоэффективность.

\section{Введение}

С промышленным развитием растет потребление электрической и тепловой энергии, основным источником которой являются ископаемые ресурсы [1-3]. Наибольшая доля приходится на уголь (при его сжигании вырабатывается около 38 \% всей электрической энергии в мире [4]). Но в то же время негативное воздействие на окружающую среду антропогенных и парниковых газов (оксидов серы $\left(\mathrm{SO}_{\mathrm{x}}\right)$, азота $\left(\mathrm{NO}_{\mathrm{x}}\right)$ и углекислого газа $\left.\left(\mathrm{CO}_{2}\right)\right)$ [5], образующихся при сжигании углей, создают предпосылки для поиска новых, менее загрязняющих атмосферу Земли топлив для тепловых электростанций. Примером такого топлива является смесь угля и биомассы. Последняя имеет низкое содержание серы и азота [6]. По результатам многочисленных исследований (например, [7-9]) установлено, что при совместном сжигании биомассы с углем существенно снижаются выбросы $\mathrm{SO}_{\mathrm{x}}$ и $\mathrm{NO}_{\mathrm{x}}$ [10-12].

Другими перспективными топливами, по экологическим критериям, являются смеси на основе воды и угля. Такую смесь в настоящее время называют водоугольным топливом (ВУТ). В работе [13] показано, что при сжигании ВУТ оксиды азота и серы снижаются практически в 2 и 4 раза, соответственно. Из теории формирования оксидов азота [14] известно, что основным источником $\mathrm{NO}_{\text {х }}$ является атомарный азот, содержащийся в воздухе (окислителе). При относительно высоких температурах среды в зоне горения протекает ряд реакций [15], основные из которых: $\mathrm{N}_{2}+\mathrm{O}_{2} \rightarrow 2 \mathrm{~N}+2 \mathrm{O} \rightarrow 2 \mathrm{NO}$. При сжигании водосодержащих топлив большое количество тепла затрачивается на парообразование, по этой причине температура в зоне горения факела существенно уменьшается по сравнению с горением пылевидного угля. В результате значительно снижается интенсивность образования $\mathrm{NO}_{\mathrm{x}}$. Кроме того, при взаимодействии водяного пара и углерода кокса $\left(\mathrm{C}+\mathrm{H}_{2} \mathrm{O} \rightarrow \mathrm{CO}+\mathrm{H}_{2}\right)$ образуются оксид углерода и водород, которые выступают в качестве восстановителя $\mathrm{NO}_{\mathrm{x}}$ и $\mathrm{SO}_{\mathrm{x}}$ при термохимическом взаимодействии $\left(\mathrm{NO}_{\mathrm{x}}+\mathrm{H}_{2} / \mathrm{CO} \rightarrow \mathrm{N}_{2}+\mathrm{H}_{2} \mathrm{O} / \mathrm{CO}_{2}\right.$; $\left.\mathrm{SO}_{\mathrm{x}}+\mathrm{H}_{2} / \mathrm{CO} \rightarrow \mathrm{S}+\mathrm{H}_{2} \mathrm{O} / \mathrm{CO}_{2}\right)$ [15].

В связи с вышеизложенным исследования водоугольных топлив, как экспериментальные [16, 17], так и теоретические [18], в последние годы проводятся достаточно интенсивно во многих государствах (особенно в России). Но водоугольные суспензии пока не нашли широкого применения в энергетике в связи с тем, что в таких топливах содержание воды должно составлять от 50 до $60 \%$ по массе [15]. Поэтому зна- 
чительная теплота затрачивается на термическую подготовку топлива (прогрев и выход паров воды из топливной частицы) и времена задержки зажигания столь обводнённых топлив могут достигать 30 с [19]. Одним из путей снижения значений $t_{\text {ign }}$ водоугольных суспензий является добавление различного рода добавок, ускоряющих процесс зажигания. В [20] с целью увеличения реакционной способности топлива в фильтр-КЕК добавлялись отходы нефтепереработки. Как известно [21], при сжигании нефтепродуктов образуется достаточно много антропогенных газов. По результатам экспериментов [20] установлено, что добавление в состав топлива отходов лесопиления приводит к снижению антропогенных выбросов.

В то же время на лесопокрытых территориях многих государств есть большие запасы отходов жизнедеятельности леса (опавшая листва, хвоя, ветки, сухостои и т. п.), так называемый лесной горючий материал (ЛГМ). Последний, с одной стороны, является источником повышенной опасности в лесах (в сухую погоду возможно возникновение лесных пожаров вследствие природных или техногенных процессов), с другой стороны - это горючие вещества, которые могут в определенной степени заменить (или дополнить) природные ресурсы (газ и уголь), использующиеся в качестве топлива тепловых электростанций. Известны примеры (достаточно многочисленные) использования различных видов биомассы в качестве добавки к углям (например, [22]). Соответственно, внедрение лесного горючего материала в энергетику позволит решить ряд проблем. Совместное сжигание биомассы и угля позволит снизить вероятность возникновения лесных пожаров путем использования отходов жизнедеятельности леса в энергетике. Также сжигание ЛГМ совместно с углем позволит решить и экологические проблемы. Но возможность использования лесных горючих материалов в качестве значимой добавки к топливам угольных ТЭС ранее не изучалась, хотя достаточно очевидно, что времена задержки зажигания ЛГМ в обычном состоянии (даже без измельчения) малы, а теплотворная способность достаточно велика. Поэтому становится актуальным использование лесного горючего материала в качестве ускоряющей процесс зажигания добавки к водоугольным топливам.

\section{Методики проведения экспериментов и подготовки топлива}

С целью подготовки топлива для экспериментальных исследований проводился ряд технологических операций. В качестве лесного горючего материала использовалась хвоя ели, которая высушивалась в сушильном шкафу в течении 120 минут при температуре 373 К. Затем лесной горючий материал измельчался в дисковой мельнице и просеивался через вибросито с размером ячейки 100 мкм. Крупнокусковой каменный уголь (размером 80..100 мм) измельчался в щековой дробилке до фракции частиц $\delta \approx 30$ мм. Далее угольная крошка размалывалась в шаровой мельнице до пылевидного состояния $\delta \leq 90$ мкм. Окончательный этап - смешение каменного угля и древесной биомассы с водой в соответствии с заданными массовыми соотношениями в гомогенизаторе. Суспензия доводилась до гомогенного (однородного) состояния в процессе перемешивания в течение 60 минут.

Состав исследуемых топливных композиций представлен в табл. 1.

Таблица 1. Состав исследуемых топливных композиций

Table 1. Structure of the studied fuel compositions

\begin{tabular}{|c|c|c|c|c|}
\hline \multicolumn{2}{|c|}{ Уголь/Coal } & \multicolumn{2}{|c|}{ Биомасса/Biomass } & Вода/Water \\
\hline $\begin{array}{c}\text { Марка } \\
\text { Brand }\end{array}$ & $\begin{array}{c}\text { Содержание/ } \\
\text { Content, \% }\end{array}$ & $\begin{array}{c}\text { Содержание/ } \\
\text { Content, \% }\end{array}$ & $\begin{array}{c}\text { Вид } \\
\text { Species }\end{array}$ & $\begin{array}{c}\text { Содержание/ } \\
\text { Content, \% }\end{array}$ \\
\cline { 1 - 3 } $\begin{array}{c}\text { «Toщий» (T) } \\
\text { Subanthracite } \\
\text { (SA) }\end{array}$ & 35 & 15 & $\begin{array}{c}\text { Еловая } \\
\text { хвоя } \\
\text { Spruce } \\
\text { needles }\end{array}$ & \multirow{2}{*}{50} \\
\cline { 2 - 3 } & 45 & 5 & 0 & 50 \\
\hline
\end{tabular}

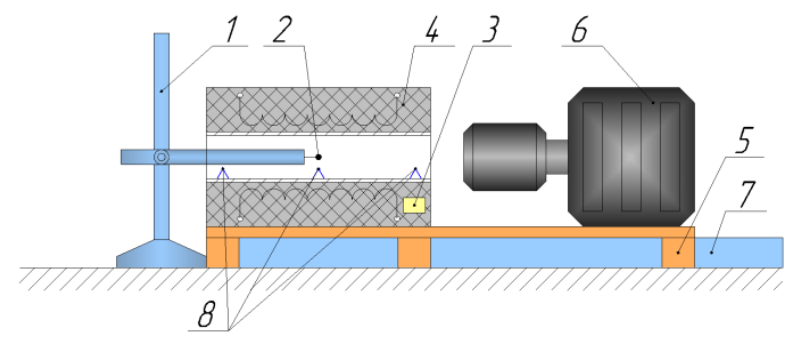

Pис. 1. Экспериментальный стенд: 1 - держатель капли; 2 - капля топлива; 3 - регулятор температуры; 4 - высокотемпературная печь; 5 - подвижная площадка; 6 - высокоскоростная видеокамера; 7 - направляющая подвижной площадки; 8- термопары

Fig. 1. Experimental stand: 1 - the drop holder; 2 - the fuel drop; 3 - the temperature controller; 4 - the hightemperature furnace; 5 - the mobile platform; 6 the high-speed video camera; 7 - the mobile platform guide; 8 - the thermocouples

На рис. 1 приведена схема экспериментальной установки, основными элементами которой являются высокотемпературная трубчатая печь и высокоскоростная видеокамера Photron FASTCAM CA4, которая установлена на подвижной каретке. Печь представляет собой полый керамический цилиндр, поделенный на три зоны нагрева с независимым управлением в каждой. Температура окислителя (воздуха) внутри печи контролировалась хромель-алюмелевой термопарой. На внешней поверхности цилиндра закреплялся термоэлектрический нагреватель. Вся система нагрева теплоизолирована минеральной ватой. Во избежание тепловых потерь торцы печи закрывались жаростойкими заглушками, которые убирались в момент, предшествующий вводу капли топлива, зафиксированной на металлическом держателе, в полость трубчатой печи. Следует отметить, что капля на держателе принимала форму вытянутого по нормали к поверхности держателя эллипсоида. Такая геометрия, как показали результаты экспериментов [23, 24], является одним из многих вариантов форм в реальной практике движения капель жидкости через высокотемпературную газовую среду. Поэтому форма капли 
после ее деформации на держателе является одной из достаточно типичных, и результаты экспериментов можно оценивать как представительные. Температура воздуха $\left(\mathrm{T}_{\mathrm{g}}\right)$ в печи соответствовала условиям топочных камер реальных котельных агрегатов и составляла от 873 до 1273 К [25]. Процессы зажигания и термической подготовки капель топлив фиксировала высокоскоростная видеокамера Photron FASTCAM CA4 (скорость видеосъемки до 20000 кад/с). За время задержки зажигания $\left(\mathrm{t}_{\mathrm{ign}}\right)$ принимался период от начала теплового воздействия до зажигания (появление первого пламени).

Для капель каждой смеси в одинаковых условиях проводилась серия экспериментов не менее чем из 10 опытов. По результатам проведенных исследований определялись средние значения $\mathrm{t}_{\mathrm{ign}}$. После этого рассчитывались среднеквадратичные отклонения каждого отдельного результата измерения и доверительные интервалы $\Delta \mathrm{t}_{\text {ign }}$ времени задержки зажигания при идентичных условиях нагрева. После отбраковки промахов определялся доверительный интервал и времена задержки зажигания при идентичных условиях нагрева. Значения $\Delta \mathrm{t}_{\mathrm{ign}}$ при доверительной вероятности 0,95 составляли не более $\pm 7,2 \%$.

\section{Результаты экспериментов}

На рис. 2 представлен внешний вид исследуемых смесей перед экспериментами. Установлено, что с увеличением доли биомассы в топливе увеличивается и адсорбционная способность смесей. Так, например, при уменьшении массовой концентрации угля и соответствующем увеличении доли лесного горючего материала в составе топлива (выше 5 \%) водоугольная суспензия теряет свою однородность (рис. 2). По этой причине распыл такого существенно неоднородного топлива может привести к забиванию форсунок или системы подачи топлива, а также абразивному износу горелок. Соответственно для сжигания водоугольной суспензии с высоким содержанием биомассы необходимы специальные технологии топливоподачи.
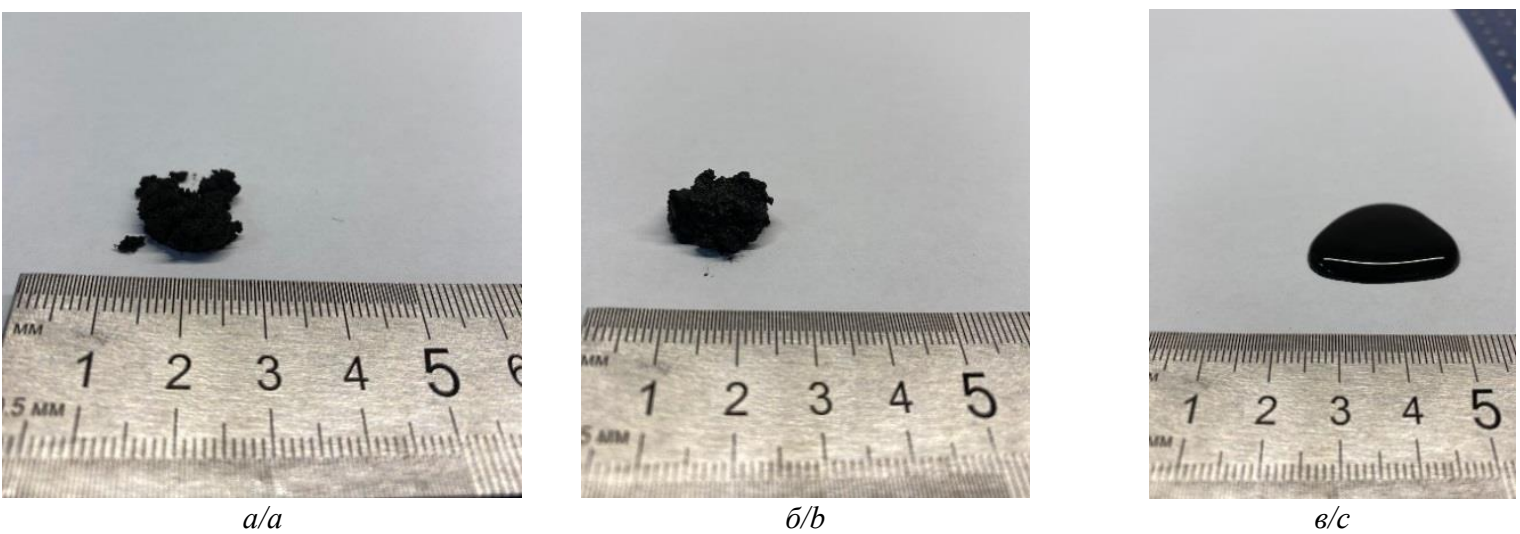

Pис. 2. Внечний вид водоугольного топлива при различных концентрациях биомассы: а) 15 \% лесного горючего материала; б) $10 \%$ лесного горючего материала; в) $5 \%$ лесного горючего материала

Fig. 2. Appearance of water-coal fuel at different biomass concentrations: a) $15 \%$ of forest combustible fuel; b) $10 \%$ of forest combustible fuel; c) $5 \%$ of forest combustible fuel

На рис. 3 представлены кадры типичной видеограммы процесса нагрева капель и последующего воспламенения частиц Био-ВУТ. Анализ кадров показывает, что весь период термической подготовки и зажигания капли топлива можно разделить на ряд последовательных и взаимосвязанных (переходящих одна в другую) стадий (высокая скорость видеосъёмки позволяет это сделать).

Кадр (а) соответствует началу теплового воздействия, которое инициирует процесс интенсивного испарения влаги. Завершение процесса влагоудаления характеризуется изменением цвета частицы Био-ВУТ (кадр (б)). Дальнейший ее нагрев приводит к пиролизу органической части угля и основных компонентов биомассы (лигнин, целлюлоза, гемицеллюлоза) с выходом горючих газов. Последующее повышение температуры поверхности частицы приводит к зажиганию Био-ВУТ (кадр (в)).

Анализ видеограмм проведенных экспериментов дает основания для еще нескольких выводов. Вопервых, сравнение кадров третьего и четвертого рядов (рис. 3) показывает, что ввод в водоугольную суспензию ЛГМ (при концентрации последнего 5 \%) приводит к тому, что капля такого топлива в течение всего периода индукции (до зажигания) сохраняет форму сферы в отличие от ВУТ без добавок ЛГМ (четвертый ряд, рис. 3). Во-вторых, при концентрациях биомассы свыше $5 \%$ формируется частица в форме, как правило, многогранников с множеством выступов и углов. Последние же являются концентраторами тепловых потоков, и, соответственно, зажигание частиц Био-ВУТ начинается именно в этих точках.

Также кадры (рис. 3, в) хорошо иллюстрируют газофазный механизм зажигания капель всех вариантов состава ВУТ (по концентрации ЛГМ). Зоны горения газов хорошо видны на всех четырех кадрах. При этом на кадре (в) (рис. 3) для состава ВУТ с концентрацией ЛГМ $10 \%$ хорошо видно, что зажигание летучих (продуктов пиролиза угля и ЛГМ) происходит над частицей, хотя по всей ее поверхности распределены участки, разогретые до высоких температур (скорее всего, это фрагменты лесного горючего материала, расположенные вблизи поверхности частицы). 


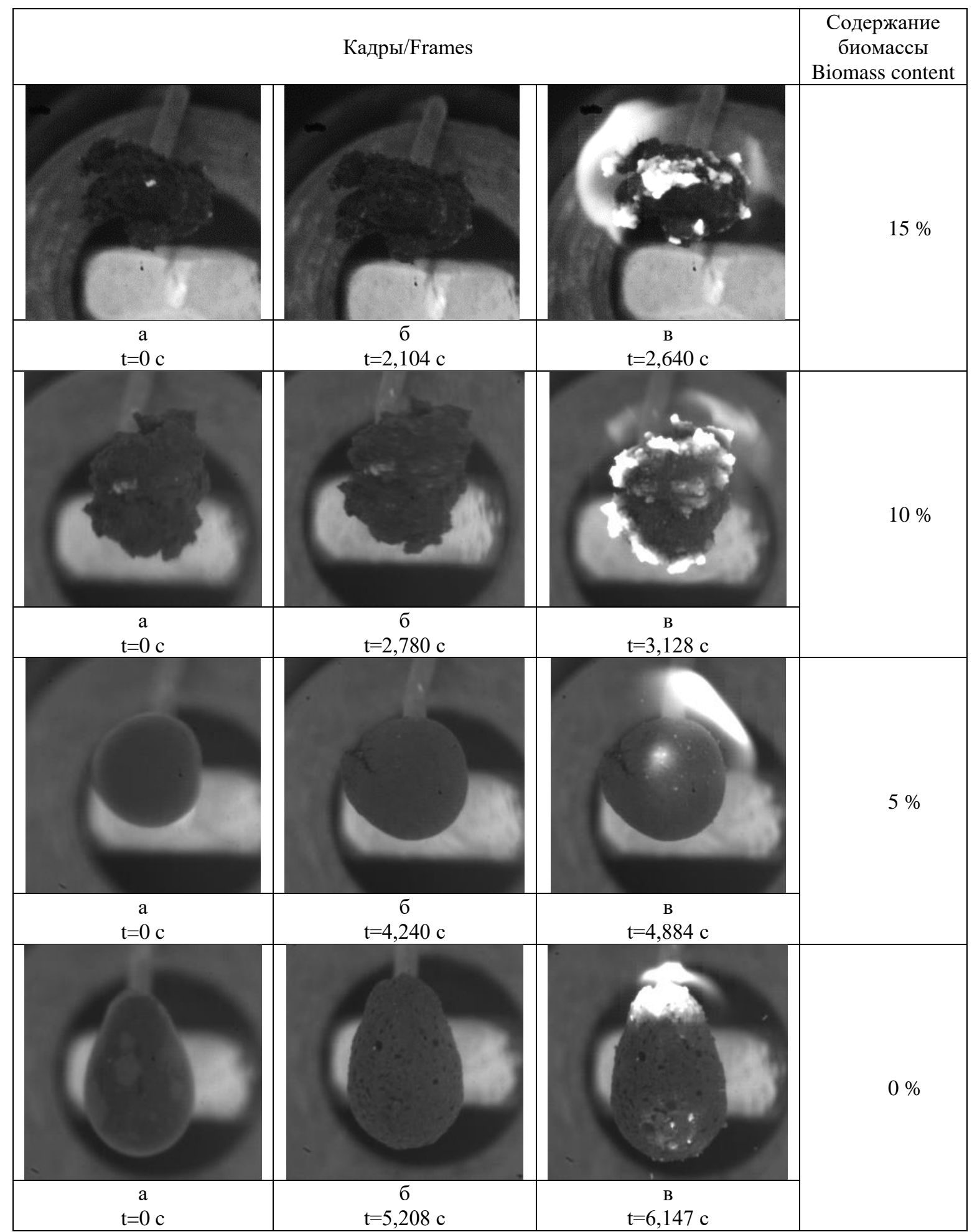

Рис. 3. Видеограмма проиесса нагрева и последующего зажигания частиць водоугольного топлива в высокотемпературной среде $\left(T_{g}=1273 \mathrm{~K}\right)$

Fig. 3. Videogram of heating and subsequent ignition of a water-coal fuel particle in a high-temperature medium $\left(T_{g}=1273 \mathrm{~K}\right)$

На рис. 4 приведены значения времен задержки зажигания одиночных капель био-водоугольного топлива (в начальный момент, а затем частиц после испарения влаги из приповерхностного слоя). Эксперименты проведены с каплями исследуемых топлив, начальные размеры которых составляли от 2 до 3,5 мм. В реальной практике после выхода из форсу- нок и распыления капель водоугольного топлива большую долю составляют капли малых (от 0,5 до 1,0 мм) размеров. Поэтому установленные в экспериментах значения $t_{\text {ign }}$ можно считать верхними оценками значений этой характеристики. В то же время в экспериментах [26] установлено, что при движении большой совокупности капель в условиях, близких к 
топочной среде, происходят процессы коагуляции капель. Большие по размерам капли поглощают малые при движении в одном направлении. Также высока вероятность коагуляции и малых капель вследствие турбулентных пульсаций в капельной струе как в продольном, так и в поперечном направлениях.
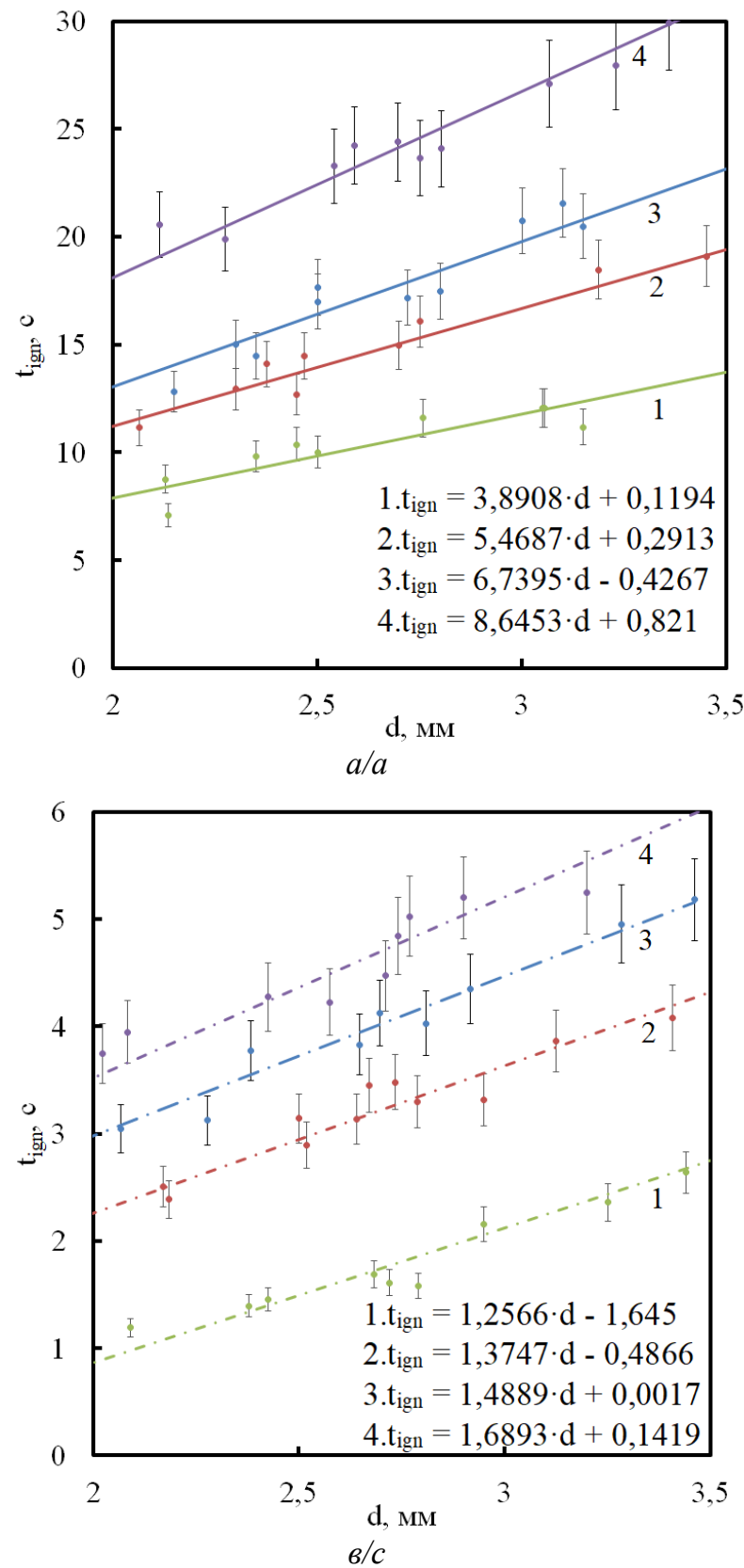

Установленные закономерности, скорее всего, обусловлены тем, что зажигание газообразных продуктов пиролиза органической части угля происходит после полного испарения всей влаги, содержащейся в топливе. Чем больше размер капли топлива, тем длительнее процесс влагоудаления и, соответственно, больше время задержки зажигания при любых температурах среды. С ростом $\mathrm{T}_{\mathrm{g}}$ скорость испарения воды растет, и значение $t_{\text {ign }}$ в результате снижается. Линейный вид зависимости $t_{\text {ign }}(d)$ может быть объяснен тем, что в период индукции протекают одновременно не-
Дробления же капель, сталкивающихся при однонаправленном движении, не происходит [26], так как их скорости сближения (если вторая «догоняет» идущую первой) малы. Поэтому диапазон изменения реальных размеров капель в проведенных экспериментах был представительным.

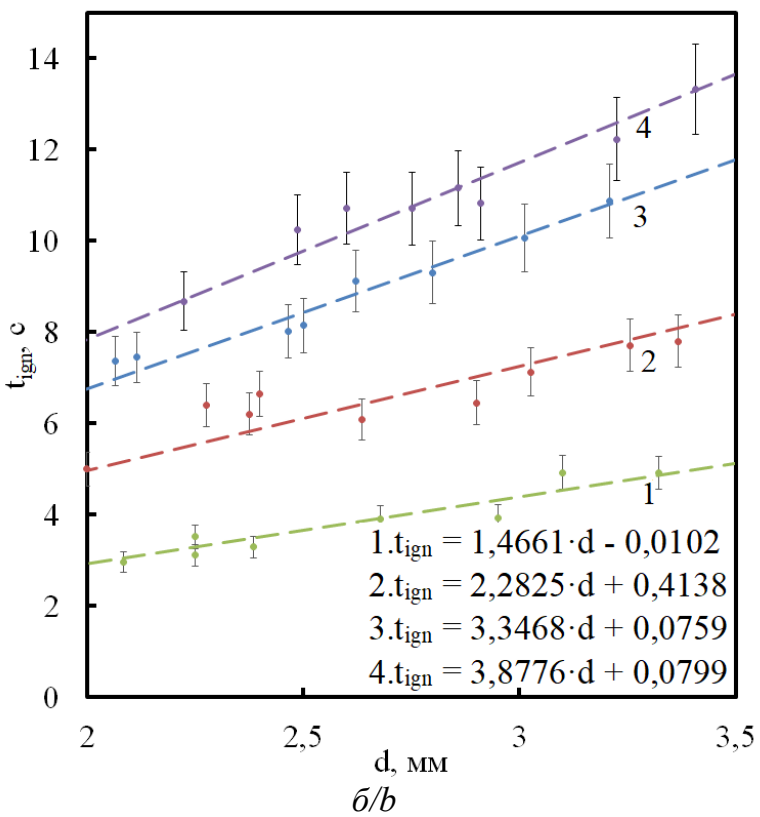

Рис. 4. Зависимости времен задержки зажигания капель водоугольных суспензий (массовая кониентрация биомассы: 1 - $15 \%, 2-10 \%, 3-5 \%, 4-$ $0 \%$ от начального размера при различных температурах окислителя $(a-873$ K, б- 1073 K, в $1273 K)$

Fig. 4. Dependences of the ignition delay times of drops of the water-coal suspensions (mass concentration of biomass: $1-15 \%, 2-10 \%, 3-5 \%, 4-0 \%$ ) on the initial size of the oxidizer at various temperatures $(a-873 K, b-1073 K, c-1273 K)$

сколько процессов, а интенсивность каждого зависит от характерного размера (условного диаметра) капли (в начальный период времени) и частицы (после испарения влаги с поверхностного слоя капли), представляющей собой двухслойную сферу (сухая оболочка и влажное ядро). Эти процессы - нагрев капли в результате излучения (в основном) и конвекции, перенос тепла в капле, теплопроводность, испарение воды, фильтрация паров воды к поверхности нагрева, вдув паров воды в слой газов в близи поверхности капли, термическое разложение угля, фильтрация 
летучих к нагреваемой поверхности, диффузия и нагрев летучих в среде окислителя, химическое взаимодействие газообразного горючего и окислителя определяют вид зависимости $\mathrm{t}_{\mathrm{ign}}(\mathrm{d})$ (каждый в большей или меньшей степени).

Основным результатом проведенных экспериментов можно считать, что смеси с добавлением ЛГМ зажигаются значительно быстрее (для смеси с добавлением 15 \% биомассы времена задержки зажигания уменьшаются более чем в три раза) по сравнению с ВУТ без добавления ЛГМ (рис. 4). Это обусловлено тем, что биомасса богата летучими, по сравнению с углем, в котором, как известно из теории формирования углей, содержание горючих газов меньше, чем в древесине. С увеличением массовой доли ЛГМ приток горючих газов в область вблизи поверхности частицы увеличивается, поэтому зажигание в газовой фазе происходит быстрее. Можно сделать обоснованный вывод, что биомасса является более реакционноспособным топливом [27] и в составе водоугольной смеси выступает в качестве «разгонщика» термохимической реакции зажигания частиц Био-ВУТ.

По результатам выполненных исследований можно сделать заключение о том, что введение ЛГМ в состав ВУТ приводит к существенному повышению ресурсоэффективности технологий сжигания ВУТ. При существенном снижении (в 2,5-3 раза) времен задержки зажигания капель водоугольных топлив с добавками ЛГМ снижается и общее время термической подготовки таких топлив. Соответственно, отпадает необходимость реконструкции топок паровых и

\section{СПИСОК ЛИТЕРАТУРЫ}

1. Miller B.G. Anatomy of a coal-fired power plant. Clean coa engineering technology (second edition). - Pennsylvania: Butterworth-Heinemann, 2017. $-856 \mathrm{p}$.

2. Zhao S. , Alexandroff A. Current and future struggles to eliminate coal // Energy Policy. - 2019. - V. 129. - P. 511-520.

3. Grigoli F., Herman A., Swiston A. A crude shock: explaining the short-run impact of the 2014-16 oil price decline across exporters // Energy Economics. - 2019. - V. 78. - P. 481-493.

4. BP Statistical Review of World Energy. URL https://www.bp.com/en/global/corporate/news-and-insights/pressreleases/bp-statistical-review-of-world-energy-2019.html (дата обращения 16.03.2020).

5. A review of greenhouse gas emission profiles, dynamics, and climate change mitigation efforts across the key climate change players / X. Zheng, D. Streimikiene, T. Balezentis, A. Mardani, F. Cavallaro, H. Liao // Journal of Cleaner Production. - 2019. V. 234. - P. 1113-1133.

6. Comparative study on combustion and oxy-fuel combustion environments using mixtures of coal with sugarcane bagasse and biomass sorghum bagasse by the thermogravimetric analysis / N.R. Galina, C.M. Romero Luna, G.L.A.F. Arce, I. Ávila // Journal of the Energy Institute. - 2019. - V. 92. - P. 741-754.

7. Furniture wood waste as a potential renewable energy source / A. Plis, M. Kotyczka-Morańska, M. Kopczyński, G. Łabojko // Thermal Analysis and Calorimetry. - 2016. - V. 125. - P. 1357-1371.

8. Van der Stelt M.J.C., Gerhauser H., Kiel J.H.A. Biomass upgrading by torrefaction for the production of biofuels: a review // Biomass Bioenergy. - 2011. - V. 35. - P. 3748-3762.

9. Nunes L.J.R., Matias J.C.O., Catalo J.P.S. A review on torrefied biomass pellets as a sustainable alternative to coal in power generation // Renew Sustain Energy Rev. - 2014. - V. 40. P. $153-160$

10. $\mathrm{NO}$ and $\mathrm{SO}_{2}$ emissions from combustion of raw and torrefied biomasses and their blends with lignite / J. Yanik, G. Duman, водогрейных котлов при переходе с угольного топлива на водоугольные суспензии в качестве основного топлива ТЭС. Кроме того, добавление 10-15 \% ЛГМ снижает потребление угля на тепловых электрических станциях и стоимость электроэнергии, так как цена ЛГМ определяется только затратами на сбор и транспортировку таких материалов. В результате использования лесных горючих материалов в качестве добавок к водоугольным топливам может существенно повысится ресурсоэффективность угольной теплоэнергетики. При этом ВУТ с добавлением ЛГМ во многих случаях будут иметь преимущества (технологические и экономические) даже по сравнению с топливами, которые представляют собой смесь сухих угля (основная компонента) и биомассы (вторая компонента) $[28,29]$. Такие смеси в последние годы достаточно интенсивно исследуются во многих развитых государствах.

\section{Заключение}

По результатам экспериментальных исследований установлена перспективность использования в качестве ускоряющей процесс зажигания капель биоводоугольного топлива добавки ЛГМ. Показано, что добавление 15 \% биомассы по массе в состав водоугольного топлива позволяет уменьшить времена задержки зажигания более чем в три раза по сравнению с водоугольной суспензией без добавления биомассы.

Исследование выполнено за счет гранта Российского научного фонда (проект № 18-79-10015).

O. Karlström, A. Brink // Journal of Environmental Management. 2018. - V. 227. - P. 155-161.

11. Vicente E.D., Alves C.A. An overview of particulate emissions from residential biomass combustion // Atmospheric Research. 2018. - V. 199. - P. 159-185.

12. Detailed Modeling of NOx and SOx formation in Co-combustion of coal and biomass with reduced kinetics / X. Wei, X. Guo, S. Li, X. Han, U. Schnell, G. Scheffknecht, B. Risio // Energy \&Fuels. 2012. - V. 26. - P. 3117-3124.

13. The prospects of burning coal and oil processing waste in slurry, gel, and solid state / K. Vershinina, G. Nyashina, V. Dorokhov, N. Shlegel // Applied Thermal Engineering. - 2019. - V. 156. P. 51-62.

14. Interaction between volatile- $\mathrm{N}$ and char- $\mathrm{N}$ and their contributions to fuel-NO during pulverized coal combustion in $\mathrm{O} 2 / \mathrm{CO} 2$ atmosphere at high temperature / X. Wu, W. Fan, P. Ren, J. Chen, Z. Liu, P. Shen // Fuel. - 2019. - V. 255. - P. 1-15.

15. Долинский А.А., Халатов А.А. Водоугольное топливо: перспективы использования в теплоэнергетике и жилищнокоммунальном секторе // Пром. теплотехника. - 2007. T. 29. - № 5. - C. 70-79.

16. Antonov D.V., Kuznetsov G.V., Strizhak P.A. Comparison of the characteristics of micro-explosion and ignition of two-fluid waterbased droplets, emulsions and suspensions, moving in the hightemperature oxidizer medium // Acta Astronautica. - 2019. V. 160 . - P. 258-269.

17. Antonov D.V., Kuznetsov G.V., Strizhak P.A. Differences of twocomponent droplets breakup at the high temperatures // Journal of the Energy Institute. - 2020. - V. 93. - P. 351-366.

18. Effect of high-temperature gas flow on ignition of the water-coal fuel particles / V. Salomatov, G. Kuznetsov, S. Syrodoy, N. Gutareva // Combustion and Flame. - 2019. - V. 203. - P. 375-385.

19. The influence of the structure heterogeneity on the characteristics and conditions of the coal-water fuel particles ignition in high temperature environment / S.V. Syrodoy, G.V. Kuznetsov, 
A.V. Zhakharevich, N.Y. Gutareva, V.V. Salomatov // Combustion and Flame. - 2017. - V. 180. - P. 196-206.

20. Sawdust as ignition intensifier of coal water slurries containing petrochemicals / K.Yu. Vershinina, G.V. Kuznetsov, P.A. Strizhak // Energy. - 2017. - V. 140. - P. 69-77.

21. Liu X., Luo Z., Yu C. Effect of limestone on the emission of NO during petroleum coke combustion // Fuel. - 2018. - V. 224. P. $1-9$.

22. Conditions and characteristics of mixed fuel granules ignition based on coal and finely dispersed wood / G.V. Kuznetsov, S.A. Yankovsky, A.A. Tolokolnikov, A.V. Zenkov, I.V. Cherednik // Energy. - 2020. - V. 194. - P. 1-9.

23. Volkov R.S., Kuznetsov G.V., Strizhak P.A. Influence of the initial parameters of spray water on its motion through a counter flow of high-temperature gases // Technical Physics. - 2014. V. 59. - P. 959-967.

24. Kuznetsov G.V., Strizhak P.A. The motion of a manifold of finely dispersed liquid droplets in the counterflow of high-temperature gases // Technical Physics Letters. - 2014. - V. 40. - P. 499-502.

25. Aerodynamic processes and heat exchange in the furnace of a steam boiler with a secondary emitter / A. Redko, R. Dzhyoiev,
A. Davidenko, A. Pavlovskaya, S. Pavlovskiy, I. Redko, N. Kulikova, O. Redko // Alexandria Engineering Journal. 2019. - V. 58. - P. 89-101.

26. Volkov R.S., Kuznetsov G.V., Strizhak P.A. Evaporation of two liquid droplets moving sequentially through high-temperature combustion products // Thermophysics and Aeromechanics. 2014. - V. 21 (2). - P. 255-258.

27. Thermal behaviour and kinetics of coal/biomass blends during cocombustion / M.V. Gil, D. Casal, C. Pevida, J.J. Pis, F. Rubiera // Bioresource Technology. - 2010. - V. 101. - P. 5601-5608.

28. Kuznetsov G.V., Yankovskii S.A. Conditions and characteristics in ignition of composite fuels based on coal with the addition of wood // Thermal Engineering. - 2019. - V. 66 (2). - P. 133-137.

29. Ignition of the wood biomass particles under conditions of nearsurface fragmentation of the fuel layer / G.V. Kuznetsov, S.V. Syrodoy, N.Y. Gutareva, A.A. Kostoreva, Zh.A. Kostoreva // Fuel. - 2019. - V. 252. - P. 19-36.

Поступила 23.03.2020 2.

\section{Информация об авторах}

Малышев Д.Ю., аспирант Инженерной школы энергетики НОЦ И.Н. Бутакова Национального исследовательского Томского политехнического университета.

Cыродой C.B., кандидат технических наук, научный сотрудник Инженерной школы энергетики НОЦ И.Н. Бутакова Национального исследовательского Томского политехнического университета. 
УДК 536.468

\title{
JUSTIFICATION OF RESOURCE EFFICIENCY OF THE TECHNOLOGIES FOR BURNING WATER-COAL FUELS WITH BIOMASS ADDITIVES
}

\author{
Dmitry Yu. Malyshev1, \\ dmitry.mlv@gmail.com \\ Semen V. Syrodoy', \\ ssyrodoy@yandex.ru \\ ${ }^{1}$ National Research Tomsk Polytechnic University, \\ 30, Lenin avenue, Tomsk, 634050, Russia.
}

One of the most pressing problems of our time is environmental safety. The pollution of the Earth's atmosphere in recent decades is caused by work at thermal power plants burning coal in the territories of the most popular states (USA, China, India, etc.). The use of a new class of fuel - bio-water-coal - can significantly reduce emissions of man-made gases, generated during combustion of coal in the furnaces of steam and hot water boilers, into the Earth's atmosphere, expand the raw materials base of the modern thermal power plants and increase the resource efficiency of coal-fired power systems.

The aim of the research is to study the influence of the concentration composition on the integral characteristics of ignition of the drops of the biohydrocarbon fuels under high-temperature heating.

Object: a water-coal suspension based on brand T coal, water and spruce needles. In the course of the experimental studies, the following mass concentrations of coal and biomass were considered 50/0 \%, 45/5\%, 40/10\%, 35/15\%, respectively (50 \% - water).

Method: experimental determination using the high-speed Photron FASTCAM CA4 video camera of the temporal characteristics of ignition of drops of the bio-water-coal suspensions under conditions corresponding to the heating intensity of the combustion chambers of the steam and hot water boilers; registration of medium temperature using chromel-alumel thermocouples.

Results. The effect of mass concentration of forest combustible material on ignition delay times (tign) of water-coal fuels has been established. It was shown that when the fuel composition contains $15 \%$ of biomass, the ignition delay times are reduced by more than three times compared with water-coal fuel without adding biomass at relatively low temperatures of the combustion medium. The results of experimental studies are also the basis for development of combustion models of liquid composite fuels.

\section{Key words:}

Bio-water-coal fuel, biomass, forest combustible fuel, ignition, ignition delay time, experiment, energy efficiency.

The research was carried out due to the grant of the Russian Science foundation (project no. 18-79-10015).

\section{REFERENCES}

1. Miller B.G. Anatomy of a coal-fired power plant. Clean coal engineering technology (second edition). Pennsylvania, Butterworth-Heinemann, 2017.856 p.

2. Zhao S., Alexandroff A. Current and future struggles to eliminate coal. Energy Policy, 2019, vol. 129, pp. 511-520.

3. Grigoli F., Herman A., Swiston A. A crude shock: explaining the short-run impact of the 2014-16 oil price decline across exporters. Energy Economics, 2019, vol. 78, pp. 481-493.

4. BP Statistical Review of World Energy. Available at: https://www.bp.com/en/global/corporate/news-and-insights/pressreleases/bp-statistical-review-of-world-energy-2019.html (accessed 16 March 2020)

5. Zheng X., Streimikiene D., Balezentis T., Mardani A., Cavallaro F., Liao $\mathrm{H}$. A review of greenhouse gas emission profiles, dynamics, and climate change mitigation efforts across the key climate change players. Journal of Cleaner Production, 2019, vol. 234 pp. 1113-1133.

6. Galina N.R., Romero Luna C.M., Arce G.L.A.F., Ávila I. Comparative study on combustion and oxy-fuel combustion environments using mixtures of coal with sugarcane bagasse and biomass sorghum bagasse by the thermogravimetric analysis. Journal of the Energy Institute, 2019, vol. 92, pp. 741-754.

7. Plis A., Kotyczka-Morańska M., Kopczyński M., Łabojko G. Furniture wood waste as a potential renewable energy source. Thermal Analysis and Calorimetry, 2016, vol. 125, pp. 1357-1371

8. Van der Stelt M.J.C., Gerhauser H., Kiel J.H.A. Biomass upgrading by torrefaction for the production of biofuels: a review. Biomass Bioenergy, 2011, vol. 35, pp. 3748-3762.

9. Nunes L.J.R., Matias J.C.O., Catalo J.P.S. A review on torrefied biomass pellets as a sustainable alternative to coal in power generation. Renew Sustain Energy Rev., 2014, vol. 40, pp. 153160

10. Yanik J., Duman G., Karlström O., Brink A. NO and $\mathrm{SO} 2$ emissions from combustion of raw and torrefied biomasses and their blends with lignite. Journal of Environmental Management, 2018, vol. 227, pp. 155-161.

11. Vicente E.D., Alves C.A. An overview of particulate emissions from residential biomass combustion. Atmospheric Research, 2018, vol. 199, pp. 159-185.

12. Wei X., Guo X., Li S., Han X., Schnell U., Scheffknecht G., Risio B. Detailed Modeling of NOx and SOx Formation in Cocombustion of Coal and Biomass with Reduced Kinetics. Energy \&Fuels, 2012, vol. 26, pp. 3117-3124.

13. Vershinina K., Nyashina G., Dorokhov V., Shlegel N. The prospects of burning coal and oil processing waste in slurry, gel, and solid state. Applied Thermal Engineering, 2019, vol. 156, pp. 51-62.

14. Wu X., Fan W., Ren P., Chen J., Liu Z., Shen P. Interaction between volatile-N and char-N and their contributions to fuel-NO during pulverized coal combustion in $\mathrm{O} 2 / \mathrm{CO} 2$ atmosphere at high temperature. Fuel, 2019, vol. 255, pp. 1-15.

15. Dolinsky A.A., Khalatov A.A. Coal-water fuels: prospects for use in the power system and housing and communal services. Industrial heat engineering, 2007, vol. 29, no. 5, pp. 70-79.

16. Antonov D.V., Kuznetsov G.V., Strizhak P.A Comparison of the characteristics of micro-explosion and ignition of two-fluid waterbased droplets, emulsions and suspensions, moving in the hightemperature oxidizer medium. Acta Astronautica, 2019, vol. 160, pp. 258-269.

17. Antonov D.V., Kuznetsov G.V., Strizhak P.A Differences of twocomponent droplets breakup at the high temperatures. Journal of the Energy Institute, 2020, vol. 93, pp. 351-366. 
18. Salomatov V., Kuznetsov G., Syrodoy S., Gutareva N. Effect of high-temperature gas flow on ignition of the water-coal fuel particles. Combustion and Flame, 2019, vol. 203, pp. 375-385.

19. Syrodoy S.V., Kuznetsov G.V., Zhakharevich A.V., Gutareva N.Y., Salomatov V.V. The influence of the structure heterogeneity on the characteristics and conditions of the coalwater fuel particles ignition in high temperature environment. Combustion and Flame, 2017, vol. 180, pp. 196-206.

20. Vershinina K.Yu., Kuznetsov G.V., Strizhak P.A. Sawdust as ignition intensifier of coal water slurries containing petrochemicals. Energy, 2017, vol. 140, pp. 69-77.

21. Liu X., Luo Z., Yu C. Effect of limestone on the emission of NO during petroleum coke combustion. Fuel, 2018, vol. 224, pp. 1-9.

22. Kuznetsov G.V., Yankovsky S.A., Tolokolnikov A.A. Zenkov A.V., Cherednik I.V. Conditions and characteristics of mixed fuel granules ignition based on coal and finely dispersed wood. Energy, 2020, vol. 194, pp. 1-9.

23. Volkov R.S., Kuznetsov G.V., Strizhak P.A. Influence of the initial parameters of spray water on its motion through a counter flow of high-temperature gases. Technical Physics, 2014, vol. 59, pp. 959-967.

24. Kuznetsov G.V., Strizhak P.A. The motion of a manifold of finely dispersed liquid droplets in the counterflow of high-temperature gases. Technical Physics Letters, 2014, vol. 40, pp. 499-502.
25. Redko A., Dzhyoiev R., Davidenko A., Pavlovskaya A., Pavlovskiy S., Redko I., Kulikova N., Redko O. Aerodynamic processes and heat exchange in the furnace of a steam boiler with a secondary emitter. Alexandria Engineering Journal, 2019, vol. 58, pp. 89-101.

26. Volkov R.S., Kuznetsov G.V., Strizhak P.A. Evaporation of two liquid droplets moving sequentially through high-temperature combustion products. Thermophysics and Aeromechanics, 2014, vol. 21 (2), pp. 255-258.

27. Gil M.V., Casal D., Pevida C., Pis J.J., Rubiera F. Thermal behaviour and kinetics of coal/biomass blends during cocombustion. Bioresource Technology, 2010, vol. 101, pp. 56015608.

28. Kuznetsov G.V., Yankovskii S.A. Conditions and characteristics in ignition of composite fuels based on coal with the addition of wood. Thermal Engineering, 2019, vol. 66 (2), pp. 133-137.

29. Kuznetsov G.V., Syrodoy S.V., Gutareva N.Y., Kostoreva A.A., Kostoreva Zh.A. Ignition of the wood biomass particles under conditions of near-surface fragmentation of the fuel layer. Fuel, 2019, vol. 252, pp. 19-36.

Received: 23 March 2020.

\section{Information about the authors}

Dmitry Yu. Malyshev, post-graduate student, National Research Tomsk Polytechnic University.

Semen V. Syrodoy, Cand. Sc, researcher, National Research Tomsk Polytechnic University. 